\title{
Precise margins and a very smooth surface
}

CEREC Primemill, Dentsply Sirona’s new grinding and milling machine, ensures producing impressive restorations with precise margins and a very smooth surface which results from the high-speed-setup with two spindles and four motors. CEREC Primemill features a powerful 7-inch touch interface, an integrated camera for scanning blocks with compatible data matrix code and an RFID scanner for reading tool information. It also works with a wide range of materials. The new design offers significantly smoother operation.

When developing the new CEREC Primemill, special attention was paid to its user-friendliness: the large touch interface guides the user through all workflow processes. Each workflow step is displayed in order and shows, for example, which tools are used for the selected material and machining option. The tools are outfitted with a colour code depending on the material to be processed and are therefore easy to distinguish. Each tool also contains a small radio frequency identification (RFID) tag that can be read by an integrated scanner in the CEREC Primemill. The machine informs the user about the tool's status and if or when it should be replaced with a new one. The new user guidance makes it even easier to delegate the operation of the machine.

For additional convenience, material blocks with a compatible data matrix code can be scanned with the integrated camera. With this the block information including

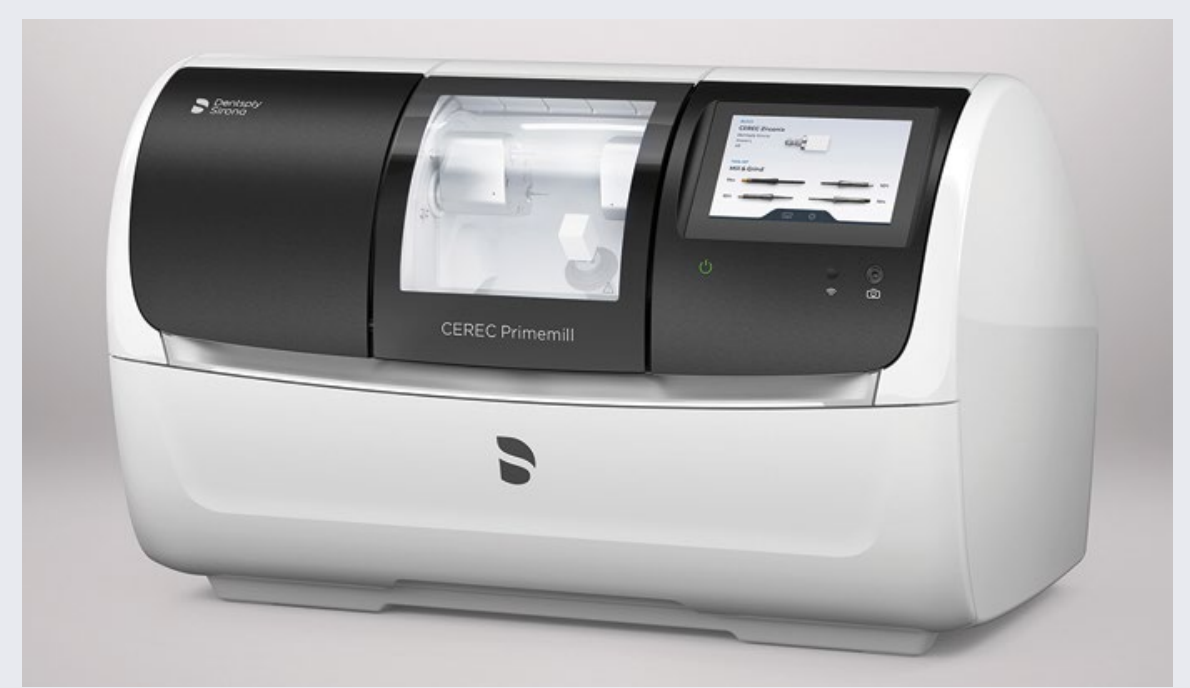

type, size, colour and zirconia enlargement factor are recorded. The unit's LED light strip also informs the user about the unit's status including a moving blue progress bar which changes to green when the manufacturing process is finished. In addition, the interface guides the user through routine maintenance procedures and thus facilitates the easy upkeep of CEREC Primemill.

With CEREC Primemill, restorations, especially those made of zirconia, can be milled even faster thanks to new tools and improved technology. The time required to fabricate a zirconia crown has been reduced by more than half: it can be cut from around 10-12 minutes to as little as five minutes using our new Super Fast mode.

The results speak for themselves. Using newly developed, very fine tools $(0.5 \mathrm{~mm})$ in the Extra Fine milling mode, the unit achieves a high level of detail for occlusal fissures as well as interdental areas on bridges, enabling users to achieve predictable, first-class results.

The entire CEREC system takes on a new dimension with CEREC Primemill. For those customers who now want to step into the chairside CAD/CAM world and want to use CAD/CAM technology in their practice, with the all-new CEREC they get a full system with great flexibility for reliable results. Users who are already successfully using CEREC in their practice will appreciate the system with the new level of speed, high level of quality, and convenience provided by CEREC Primemill.

www.dentsplysirona.com.

\section{Integrated solutions for endodontics}

On 3 February, VDW launched the VDW. FLO Endo Organizer for the global market. The multifunctional insert for drawers and equipment trolleys organises loose instruments, irrigation, obturation and post-endodontic materials to create a clear overview of the inventory. Used on a table, the organiser keeps all necessary materials tidy and readily at hand during treatment. Its 16 small, medium and large compartments are specially designed for common packaging sizes. The VDW.FLO Endo Organizer can be adapted to individual workflows with the aid of the corresponding labelling set of blank and pre-printed stickers. The VDW.FLO Endo Organizer is made of easy-to-clean, robust plastic and features a non-slip silicone underside to facilitate

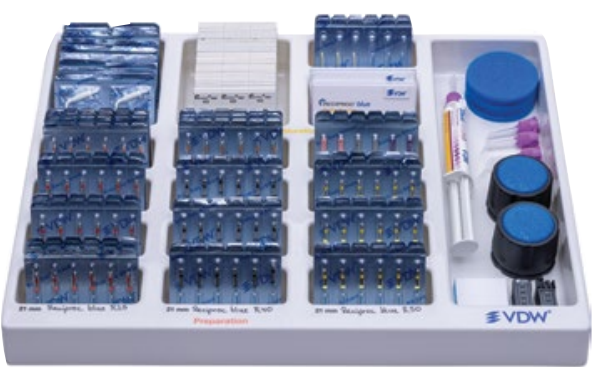

compliance with hygiene measures in practice.

For over 150 years VDW has represented experience in the development and manufacture of products for endodontics. VDW develops innovative ideas and produces optimised solutions to improve the clinical success of endodontic treatments. Today, VDW offers a holistic range of solutions covering the entire endodontic treatment spectrum, from obturation to post-endodontic care. The company also has numerous application-related advanced training programmes in endodontics.

www.vdw-dental.com/en/. 